\title{
Absence of FLT3 mutations in Iranian adult T-cell leukemia/lymphoma patients
}

\author{
Hossein Ayatollahi ${ }^{1}$, Mohammadhadi Sadeghian ${ }^{1}$, Mohammadmahdi Kooshyar $^{2}$, Abass Shirdel $^{2}$, \\ Hossein Rahimi ${ }^{2}$, Amirhossein Jafarian ${ }^{3}$, Saeedeh Ghazaey Zidanloo ${ }^{4}$, Narges Soltani ${ }^{4}$, Fatemeh Shams ${ }^{4}$, \\ Neda Motamedi Rad ${ }^{5}$, Sepideh Shakeri*4
}

\section{Abstract}

Background: Adult T cell leukemia lymphoma (ATLL) is a rare disease, significantly linked to the infection by the human T-cell lymphotropic virus 1(HTLV-1). ATLL is typically preceded by decades of clinical latency during which infected cells accumulate selectable traits leading to a malignant transformation. Amongst all the HTLV-1 infected carriers only about 3-5\% will develop ATLL. Despite the intensive attempt to improve the overall survival, ATLL remains one of worse prognosis among the hematologic malignancies. FMS like tyrosine kinase 3 internal tandem duplication (FLT3-ITD) mutations are mutations which are frequent among leukemic patients. We aimed to investigate the frequency of FLT3 mutation status in patients with acute type of ATLL which has not been studied yet.

Methods: In this case control study 38 patients with acute type of ATLL were retrospectively analyzed between February 2015 and February 2017. Forty HTLV-1 positive patients were also used as control cases. Genomic DNA was extracted according to phenolchloroform protocol and two restriction fragment length polymorphism (RFLP) PCR reactions were set up to detect FLT3/ ITD and FLT3/TKD mutations. Differences between variables were evaluated by the chi-square test and $t$ test for categorical and continuous variables, respectively. SPSS software v. 15 was used for statistical analysis. All P values were two sided and values less than 0.05 were considered to be significant.

Results: No FLT3 mutations were detected in acute type of ATLL patients. So far, not many studies have shown the frequency of FLT3 mutation in ATLL patients

Conclusion: Therefore, we conclude that although FLT3 mutations are rather unusual in the acute type of ATLL patients, but other alternative mechanisms associated with ATLL remain to be further investigated. This study was a novell project regarding the analysis of FLT3 mutation in the field of ATLL research.

Keywords: Adult T cell leukemia, Human T-cell lymphotropic virus 1, Iran

Copyright $\odot$ Iran University of Medical Sciences

Cite this article as: Ayatollahi H, Sadeghian M, Kooshyar M, Shirdel A, Rahimi H, Jafarian A, Ghazaey Zidanloo S, Soltani N, Shams F, Reseal Z, Sheikhi M, Shakeri S. Absence of FLT3 mutations in Iranian adult T-cell leukemia/lymphoma patients. Med J Islam Repub Iran. $2018(9$ June);32:47. https://doi.org/10.14196/mjiri.32.47

\section{Introduction}

Human T-cell lymphotropic virus 1 (HTLV-1) is the HIV/AIDS like virus which is endemic to certain parts of the world such as Japan, the Caribbean, West Africa, South and Central America and Caribbean area, with foci in Middle-east and Australo-Melanesia (1). One of en-

\footnotetext{
Corresponding author: Sepideh Shakeri, ssh.shakeri@gmail.com
}

1. Department of Hematology and Blood Bank, Faculty of Medicine, Cancer Molecular Pathology Research Center, Ghaem Medical Center, Mashhad University of Medical Sciences, Mashhad, Iran

2. Department of Internal Medicine, Faculty of Medicine, Ghaem Medical Center, Mashhad university of Medical sciences, Mashhad, Iran

3. Department of Pathology, Cancer Molecular Pathology Research Center, Ghaem Medical Center, Mashhad University of Medical Sciences, Mashhad, Iran

${ }^{4}$. Cancer Molecular Pathology Research Center, Ghaem Medical Center, Mashhad University of Medical Sciences, Mashhad, Iran

5. Cancer Molecular Pathology Research Center, Ghaem Medical Center, Mashhad University of Medical Sciences, Mashhad, Iran demic regions for HTLV-1 is the Middle-east (2, 3). Great Khorasan in northeastern part of Iran is reported as being an endemic region for infection by HTLV-1, with a prevalence of $1.97 \%$ (4). This retrovirus, may cause adult T-cell leukemia/lymphoma (ATLL), and HTLV-associated neu-

$\uparrow$ What is "already known" in this topic:

ATLL is a rare disease that is significantly linked to the infection by the HTLV1. FLT3 mutations are the most frequent molecular aberrations in leukemic patients. FLT3 mutations had significant prognosis in leukemia.

$\rightarrow$ What this article adds:

Because there are many ATLL patients in our regions, we decided to analysis ATLL patients for FLT3 mutations. We did not find any FLT3 mutations in ATLL patients. FLT3 mutations are found in different kind of leukemia but there are not found in ATLL patients. 
ropathies. ATLL is an unusual and aggressive T-cell lymphoma linked to HTLV-1 infection. So far, the ATLL disease could not be predicted in HTLV-1 carriers of different stages $(5,6)$. In spite of the intensive attempts to improve the overall survival, ATLL is still one of the hematologic malignancies with the poorest prognoses. It has been reported that the disease may proceed by decades of clinical latency during which infected cells accumulates selectable traits leading to a malignant transformation (7). ATLL patients are presented with a variety of clinical features, so the disease is classified into four clinical types: smoldering, chronic, acute, and lymphoma types. Smoldering and chronic type cases have an indolent clinical course and achieve long-term survival even without chemotherapy. In acute type cases, the prognosis is quite poor, despite of the developments of novel therapeutic reagents and supportive advances. Almost $4-5 \%$ of HTLV-I carriers are thought to be developing ATLL after long latency periods, generally 40-50 years. Multiple steps or multi-factorial events have been anticipated to be required for the development of cancer (8). ATLL patients may appear clinically with lymphadenopathy and hepatosplenomegaly; skin infiltrates are usual but not always present. Hypercalcemia is common, and causes severe thirst in some of the patients. Morphologically leukemic cells are abnormal: the characteristics is a large, lobulated nucleus which gives rise to the epithet "flower cell (9)."

The Fms-like tyrosine kinase 3 (FLT3) genes encode a class III receptor tyrosine kinase strongly associated with cellular proliferation and differentiation (10). FLT3 is expressed in different types of myeloid and B-lymphoid lineages, and also expressed at high levels in different types of hematologic malignancies containing $70 \%$ to $100 \%$ of acute myelogenous leukemia (AML) of all French-American-British (FAB) subtypes, B-precursor cell acute lymphoblastic leukemia (ALL), a fraction of Tcell ALL, and chronic myelogenous leukemia (CML) in lymphoid blast crisis. FLT3 mutations are among the most frequent molecular aberrations detected in AML patients. Two principal types of mutations have been recognized: internal tandem duplication mutations (ITDs) discovered in $20-25 \%$ of patients with AML and tyrosine kinase domain (TKD) point mutations, D835, happening in 5-10\%, FLT3/TKD mutations; which occurs at or near the active site, most commonly at aspartate 835 (11). In recent years, the identification of abnormal protein tyrosine kinase (TK) signaling pathways in many human cancers has led to the diagnostic and therapeutic advances (12). However, FLT3 is infrequently mutated in leukemic lymphoblasts (13). These findings illustrate a special function for FLT3 in development of multipotent stem cells as well as B cells (2). Similarly, previous studies have shown conflicting results concerning the role of FLT3 mutations in patients with AML, ALL and CML. These data indicated that
FLT3 expression may play a role in the survival or proliferation of leukemic blasts (14). Regarding to significant role of FLT3 mutation in other leukemic types this study aimed to investigate the frequency of its mutation status in patients with acute type of ATLL which has not yet been studied.

\section{Methods}

\section{Patients}

Fresh bone marrow or peripheral blood samples from 38 patients with newly diagnosed acute type of ATLL admitted to Ghaem and Emam Reza hospitals between February 2015 and February 2017 were analyzed. All patients were diagnosed according to bone marrow morphology, cytochemistry and immunophenotype. Laboratory findings in ATLL are hypercalcemia and presence of large, lobulated nucleus which named "flower cell', All patients examined by dermatologist and cases with dermatitis lesions were classified as positive group. Finally the percentage of positive cases was calculated.

The peripheral blood samples from 40 HTLV1 positive volunteers were used as control. Cases and controls were age matched. For preservation of samples and genetic analysis, all cases were given informed consent approved by Ethics Committee of Mashhad University of Medical Sciences (MUMS) according to Declaration of Helsinki.

\section{Nucleic acid isolation}

Genomic DNA was extracted using phenolchloroform- isoamyl alcohol protocol from $2 \mathrm{ml}$ fresh bone marrow or peripheral blood cells after Ficoll separation of mononuclear cells. The concentration of DNA was specified by the ratio of A260/A280 and then diluted to 1 $\mu \mathrm{g} / \mu \mathrm{l}$ with $\mathrm{ddH}_{2} \mathrm{O}(15)$.

\section{Detection of FLT3/ITD by DNA PCR}

For the FLT3/ITD mutation analysis, the following primers were used; 14 forward 5'-GCAATTTAGGTATGAAAGCCAGC-3' and 15 reverse 5'CTTTCAGCATTTTGACGGCAACC-3' (Table 1), and PCR was performed over 35 cycles of $1 \mathrm{~min}$ at $94{ }^{\circ} \mathrm{C}, 30$ $\mathrm{sec}$ at $60{ }^{\circ} \mathrm{C}$, and $90 \mathrm{sec}$ at $72{ }^{\circ} \mathrm{C}$. The following primers was also amplified the FLT3/TKD region; 17forward 5'CCGCCAGG AACGTGC-TG-3'and 17 reverse 5'GCAGCCTCACATTGCCCC-3' (Table 1), over 35 cycles of $1 \mathrm{~min}$ at $94{ }^{\circ} \mathrm{C}, 1 \mathrm{~min}$ at $66^{\circ} \mathrm{C}$, and $90 \mathrm{sec}$ at $72{ }^{\circ} \mathrm{C}$. Amplification was performed in a $20 \mu \mathrm{l}$ reaction volume with $1 \mu \mathrm{l}$ DNA $(1 \mu \mathrm{g} / \mu \mathrm{l}), 10 \mathrm{pmol}$ each primer, 10 mmoldNTP, 2.5U Ex-Taq DNA polymerase(Takara, Japan) in the buffer (10 mmol/1 Tris- $\mathrm{HCl}(\mathrm{pH} 8.3), 50$ $\mathrm{mmol} / \mathrm{l} \mathrm{KCl}$ and $1.5 \mathrm{mmol} / 1 \mathrm{MgCl} 2)$. The primers are listed in Table 1 , and PCR products were stored at $-20^{\circ} \mathrm{C}$ until post-PCR analysis. A restriction fragment length polymorphism (RFLP)--mediated PCR assay was used to

Table 1. The primer sequence and size of their bands.

\begin{tabular}{|c|c|c|c|c|}
\hline & Forward Primer & Reverse Primer & $\begin{array}{l}\text { Wild type } \\
\text { (bp) }\end{array}$ & $\begin{array}{c}\text { Mutant } \\
\text { (bp) }\end{array}$ \\
\hline FLT3-ITD & 5'-GCAATTTAG-GTATGAAAGCCAGC-3' & 5'-CTTTCAGCATTTTGACGGCAACC-3' & $328 \mathrm{bp}$ & $328+>328 \mathrm{bp}$ \\
\hline FLT3-TKD & 5'-CCGCCAGG AACGTGC-TG-3' & 5'-GCAGCCTCACATTGCCCC-3' & $46+68 b p$ & $114+46+68 b p$ \\
\hline
\end{tabular}


detect FLT3 mutations, because D835 and I836 codons encoded by the nucleotide sequence GATATC, exhibited an Eco RV restriction site. Amplified products were digested with Eco RV, which yielded 68 and 46 bp fragments. The D835 mutation showed an additional undigested band (114 bp).and electrophoresis was performed in an agarose gel (15).

ITD mutations were detected by $3 \%$ agarose gel electrophoresis of the PCR products. In a reaction of specimen heterozygous for an FLT3/ITD mutation, the fragments generated in the PCR consist of the 330-bp wild-type and the longer mutant fragments, the length increased over the wild-type because of the inserted sequence, so the increased length of the mutant sequence turns into a longer time required to complete a PCR cycle, which in turn led to the wild-type allele being amplified at a more rapid rate than the mutant sequence. The size of bands are listed in Table 1 .

This protocol usually demonstrates a wild-type band (328bp) and a larger-size band indicating the ITD mutation. D835 mutations were detected by electrophoresis of the amplified products following digestion with Eco RV. The amplified products of wild-type alleles were digested into two bands ( 68 bp and 46 bp) by Eco RV. Amplified products with D835 mutations, had undigested bands (114 bp) visualized on agarose gel electrophoresis. Inclusion of a negative control was essential to ensure complete digestion by Eco RV, therewith eliminating the possibility of false-positive results in patient samples (15). The positive and negative control are shown in Figure 1, a and b.

\section{Statistical analysis}

Differences between variables were evaluated by the chi-square test and t test for categorical and continuous variables, respectively. SPSS software v. 15 was used for statistical analysis. All $\mathrm{P}$ values were two sided and values less than 0.05 considered to be significant.

\section{Results}

\section{Clinical and laboratory characteristics of all patients}

In this research 38 ATLL patients including 24 men and 14 women with mean age of $57.2 \pm 18.4$ were entered the study. The clinical and laboratory diagnostics findings of the 38 patients and 40 HTLV1 positive carriers are summarized in Table 2. Abnormal elevation of serum lactate dehydrogenase (LDH) and calcium levels were notably elevated in all of patient with subtypes of ATLL. Skin lesions were detected in $57.9 \%$ of acute group and it was not found in control ones. Peripheral lymphadenopathy reported in $44.7 \%$ of acute group, was not reported in con-

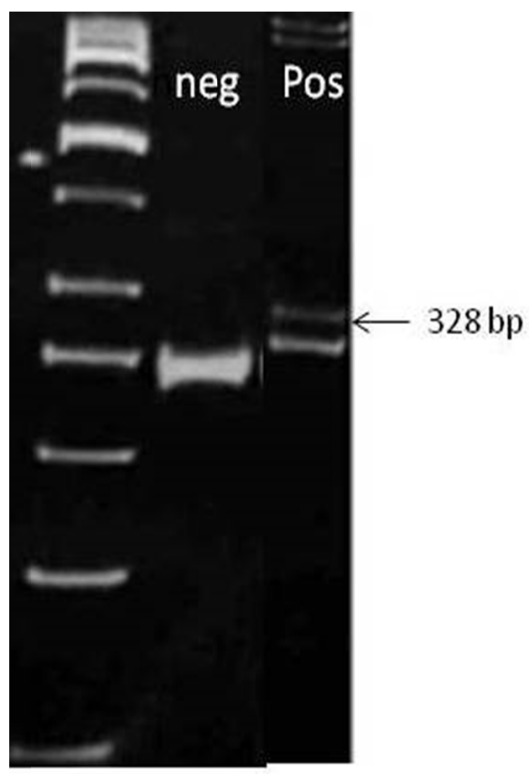

a)FLT3-ITD

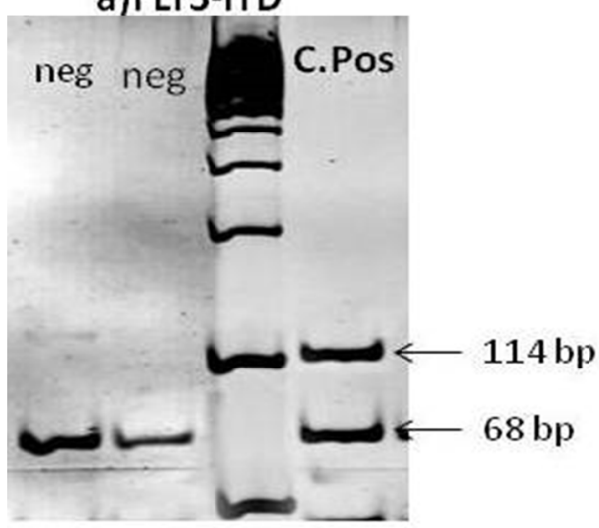

b) FLT3-TKD

Fig. 1. Detection of FLT3 mutations by genomic PCR. (a) PCR product of the FLT3/ITD mutation in the juxtamembrane domain. Products longer than normal328 bp were observed. (b) EcoRV restriction of PCR product of the FLT3/TKD mutation in tyrosine kinase domain. Products not restricted by EcoRV indicated a point mutation present at the tyrosine kinase domain $(\mathrm{C}$ : control)

trol group Statistical analysis revealed no meaningful differences between gender and studied groups.

\section{Prevalence of FLT3/ITD and FLT3/TDK mutation}

The FLT3/ITD and FLT3/TKDPCR products yielded a higher molecular weight product on a $2 \%$ agarose gel stained with ethidium bromide (Fig. 1). Both positive and negative controls were included in the PCR reaction. Al-

Table 2. Clinical and laboratory characteristics and their correlation with clinical subtypes and survival in 38 cases of acute subtype of adult T-cell leukemia/lymphoma are compared with 40 cases of HTLV1 positive volunteers.

\begin{tabular}{|c|c|c|c|c|c|}
\hline \multirow[t]{2}{*}{ Characteristic } & \multicolumn{2}{|c|}{ Acute group } & \multicolumn{2}{|c|}{ Control group } & \multirow[t]{2}{*}{$\mathrm{p}$} \\
\hline & Mean & $\mathrm{SD}$ & Mean & $\mathrm{SD}$ & \\
\hline Age,(year) Mean & 57.2 & 18.4 & 50.6 & 19.2 & 0.725 \\
\hline WBC $\left(\times 10^{6} / \mathrm{L}\right)$ Mean & 34.3 & 15.2 & 6.2 & 2.2 & 0.008 \\
\hline Serum LDH, IU $/ \mu \mathrm{L}$ Mean & 720 & 242 & 250 & 64 & 0.002 \\
\hline Absolute lymphocyte & 25.6 & 7.2 & 3.6 & 1.8 & \\
\hline Count, $\times 10^{6} / \mathrm{L}$ Mean & & & & & 0.007 \\
\hline Calcium total $(\mathrm{mmol} / \mathrm{L})$ Mean & 3.3 & 0.6 & 2.3 & 0.2 & 0.011 \\
\hline
\end{tabular}


terations in the FLT3 gene, neither FLT3/ITD nor FLT3/TKD, were not detected in 38 ATLL cases and analyzed 40 HTLV1 cases (Fig. 1). In spite of the fact that direct sequencing techniques have a relatively low sensitivity for detection of acquired mutations (roughly 20\%), the probability of false negative results in this setting was very unlikely.

\section{Discussion}

According to the high prevalence of HTLV1 in Khorasan province and our country and its association with ATLL $(16,17)$, we investigated the frequency of FLT3 mutations in acute type of ATLL patients. ATLL is an aggressive T-cell neoplasm which is resistant to variety of therapy regimens. Due to the fact that it is a rare disease, small case series have been studied. It is important to notify that HTLV1 infection is epidemic in Khorasan Razavi region, and ATLL caused by of HTLV1 infection. Although stem cell transplantation is an important strategy for treatment, newly diagnosed patients may only expect a median survival of 12 months. Possibly through better comprehension of the unique biology and pathogenesis of ATLL novel therapeutic targets could be generated (18).

Moreover, genetic instability is a frequent phenomenon in human cancers. Despite the fact that the molecular mechanisms mediating this effect are not completely understood, it has been suggested to involve either the loss of DNA repair abilities or chromosomal instability. Therefore, the HTLV-I is believed to cause the genomic instability (19).

Molecular mutations are raising importance for the diagnosis and prognosis in some type of hematological malignancies such as, CML and Follicular Lymphoma (20, 21, and 22). Some mutations have been detected to be prognostically favorable as long as some others are unfavorable (23). The description of relevant molecular markers for the determination of prognosis and for minimal residual disease (MRD) detection is of increasing importance. Molecular mutations, exclusively in normal karyotype, are becoming more important for disease classification and to assess the prognosis in many cancers.

Mutations of FLT3, one of the most constant somatic alterations in AML, have been a possible target for its molecular therapy. Many activating mutations, mostly internal ITD and rarely point mutations in the activation loop of FLT3, have been diagnosed in AML patients. Because of the high frequency of FLT3 mutations in some leukemia such as AML, concentration focuses on the development of FLT3 inhibitors for treatment of this leukemia (24). In this report, we have demonstrated that there was no FLT3 mutations in our acute type of ATLL cases. So far there is not any similar study that has investigated the prevalence of these mutations in acute type of ATLL patients, Therefore, further studies in different other populations involving larger number of patients are needed to clearly elucidate the prevalence and functional implication of the FLT3 mutations in the pathogenesis of ATLL.

Several studies have been reported some other genetic alterations correlated with ATLL. Over several years different studies have suggested central roles for JAK3/_c- associated cytokines mutations, high rate of activating mutations in the Notch gene and the TCF8 gene inactivation in ATLL pathogenesis $(23,25$, and 26).

Furthermore, the stepwise process and accumulation of chromosomal aberrations also may lead to oncogenesis, and the variation of these processes and involved genes will indicate marked variations in clinical presentation of ATLL. In the current study the cytogenetic analysis was not used to exclude the probability that unidentified repeated translocations that may cause the activation of proto oncogenes. Nevertheless, it should be noted that the possible activation of proto oncogenes is one event in the serial multistep process of carcinogenesis (27).

Although genetic and cytogenetic alterations in patients with ATLL rarely were studied, there were, however, no reports of FLT3 mutations in ATLL cases yet. In the present report, we failed to detect any mutations among our patients by genomic DNA PCR. One of the limitations in similar studies in ATLL was the small sample size with limited number of patients as this disease is relatively rare.

\section{Conclusion}

No FLT3 mutations were detected in acute type of ATLL patients, but other alternative mechanisms such as other genes may involve in the pathogenesis of the disease such as Tax and HBZ, hence for the activation of proliferation further investigation is needed.

\section{Conflict of Interests}

The authors declare that they have no competing interests.

\section{References}

1. Gessain A, Cassar O. Epidemiological aspects and world distribution of HTLV-1 infection. Frontiers in microbiology. 2012;3.388

2. Azarpazhooh MR, Hasanpour K, Ghanbari M, Rezaee SR, Mashkani B, Hedayati-Moghaddam MR, et al. Human T-lymphotropic virus type 1 prevalence in northeastern Iran, Sabzevar: an epidemiologicbased study and phylogenetic analy'sis. AIDS research and human retroviruses. 2012;28(9):1095-101.

3. Boostani R, Ardakani AM, Ashrafï H. Khorasan disease: prevalence of HTLV-I associated myelopathy/tropical spastic paraparesis (HAM/TSP) in West Azarbaijan from 2004 to 2007. Iranian Red Crescent Medical Journal. 2011;13(6):42.

4. Khameneh ZR, Sepehrvand N, Masudi S, Taghizade-Afshari A. Seroprevalence of HTLV-1 among kidney graft recipients: a singlecenter study. Exp Clin Transplant. 2010;8(2):146-9.

5. Pawson R, Mufti GJ, Pagliuca A. Management of adult T-cell leukaemia/lymphoma. Br J Haematol. 1998;100(3):453-8.

6. O'Connor OA. Getting the facts: adult T-cell leukemia/lymphoma (HTLV-1). Lymphoma Research Foundation website. http://www.lymphoma.org/atf/cf/ \{0363cdd6-51b5-427b-be48e6aF871acec9\}/htlv.pdf. Accessed June 15, 2011.

7. Rauch DA, Ratner L. Targeting HTLV-1 activation of NFкB in mouse models and ATLL patients. Viruses. 2011; 3(6):886-900.

8. Kohno T, Yamada Y, Tawara M, Takasaki Y, Kamihira S, Tomonaga $\mathrm{M}$, et al. Inactivation of p14 ARF as a key event for the progression of adult $\mathrm{T}$ cell leukemia/lymphoma. Leukemia research. 2007; 31(12):1625-32.

9. Bangham CR, Toulza F. Adult T cell Leukemia/Lymphoma: FoxP3+ Cells and the Cell-Mediated Immune Response to HTLV-1. Advances in cancer research. 2011;111:164.

10. Park SH, Chi HS, Min SK, Cho YU, Jang S, Park CJ, et al. Prognostic significance of the FLT3 ITD mutation in patients with normalkaryotype acute myeloid leukemia in relapse. The Korean journal of hematology. 2011;46(2):88-95.

11. Nazha A, Cortes J, Faderl S, Pierce S, Daver N, Kadia, et al. Acti- 
vating mutations of the FMS-like tyrosine kinase-3 internal tandem duplication (FLT3-ITD) at complete response and relapse in patients with acute myeloid leukemia. Haematologica. 2012;102(12):1-14.

12. Mead AJ, Linch DC, Hills RK, Wheatley K, Burnett AK, Gale RE. FLT3 tyrosine kinase domain mutations are biologically distinct from and have a significantly more favorable prognosis than FLT3 internal tandem duplications in patients with acute myeloid leukemia. Blood. 2007;110(4):1262-70.

13. Paietta E, Ferrando AA, Neuberg D, Bennett JM, Racevskis J, Lazarus $\mathrm{H}$, et al. Activating FLT3 mutations in CD117/KIT+ T-cell acute lymphoblastic leukemias. Blood. 2004;104(2):558-60.

14. Tsapogas P, Mooney CJ, Brown G, Rolink A. The Cytokine Flt3Ligand in Normal and Malignant Hematopoiesis. Int J Mol Sci. 2017 May $24 ; 18(6)$.

15. Wang L, Lin D, Zhang X, Chen S, Wang M, Wang J. Analysis of FLT3 internal tandem duplication and D835 mutations in Chinese acute leukemia patients. Leukemia research. 2005;29(12):1393-8.

16. Safabakhsh H, Jalalian M, Karimi G. Seroepidemiology of human T-cell lymphotropic virus type-1 (HTLV1) in Mashhad. Glob J Health Sci. 2014 May 13;6(5):99-104

17. Taylor GP. Molecular aspects of HTLV-I infection and adult T-cell leukemia/lymphoma. Journal of clinical pathology. 2007; 60(12):1392-6.

18. Levis M1. FLT3 mutations in acute myeloid leukemia: what is the best approach in 2013? Hematology Am Soc Hematol Educ Program. 2013;2013:220-6

19. Ahmadi Ghezeldasht S1, Shirdel A2, Assarehzadegan MA3, Hassannia T4, Rahimi H2, Miri R1, Rezaee SA. Human T Lymphotropic Virus Type I (HTLV-I) Oncogenesis: Molecular Aspects of Virus and Host Interactions in Pathogenesis of Adult $\mathrm{T}$ cell Leukemia/Lymphoma (ATL). Iran J Basic Med Sci. 2013;16(3):179-95.

20. Karimiani EG, Day P. Personalised treatment of haematological malignancies through systems medicine based on single molecules in single cells. Integrative Biology. 2013;5(5):759-67.

21. Sandison HE, Usher S, Karimiani EG, Ashton G, Menasce LP, Radford JA, et al. PLK1 and YY1 interaction in follicular lymphoma is associated with unfavourable outcome. Journal of clinical pathology. 2013 Jun 10:2013.

22. Verma M, Karimiani EG, Byers RJ, Rehman S, Westerhoff HV, Day PJ. Mathematical modelling of miRNA mediated BCR. ABL protein regulation in chronic myeloid leukaemia vis-a-vis therapeutic strategies. Integrative Biology. 2013;5(3):543-54.

23. Li W, Zhang L, Huang L, Mi Y, Wang J. Meta-analysis for the potential application of FLT3-TKD mutations as prognostic indicator in non-promyelocytic AML. Leukemia research. 2012;36(2):186-91.

24. Bacher U, Haferlach C, Kern W, Haferlach T, Schnittger S. Prognostic relevance of FLT3-TKD mutations in AML: the combination matters - an analysis of 3082 patients. Blood. 2008; 111(5):2527-37.

25. Pancewicz J, Taylor JM, Datta A, Baydoun HH, Waldmann TA, Hermine $\mathrm{O}$, et al. Notch signaling contributes to proliferation and tumor formation of human T-cell leukemia virus type 1-associated adult T-cell leukemia. Proceedings of the National Academy of Sciences. 2010;107(38):16619-24.

26. Hidaka T, Nakahata S, Hatakeyama K, Hamasaki M, Yamashita K, Kohno T, et al. Down-regulation of TCF8 is involved in the leukemogenesis of adult T-cell leukemia/lymphoma. Blood. 2008;112(2):38393. 